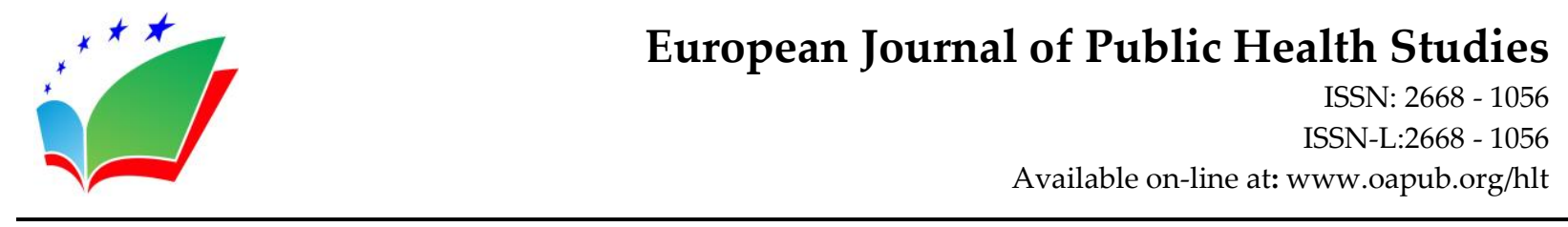

DOI: $10.46827 /$ ejphs.v4i2.105

Volume 4 | Issue 2 | 2021

\title{
EVALUATION OF VACCINATION RATES IN PATIENTS DIAGNOSED WITH COVID
}

\author{
Esra Arslan Aksu'i , \\ Demet Yalcin Kehribar ${ }^{2}$ \\ 1Samsun Training and Research Hospital, \\ Department of Chest Disease, \\ Samsun, Turkey \\ ${ }^{2}$ Ondokuz Mayis University, \\ Medical Faculty, \\ Department of Internal Medicine, \\ Samsun, Turkey
}

\begin{abstract}
:
The Covid-19 pandemic has caused many people to die all over the world. Isolation rules are not enough to control the epidemic, and the most important solution for returning to normal life is vaccination. In our study, data on vaccination rates and clinical courses in hospitalized patients were examined and results regarding the effectiveness of the vaccine were obtained. Vaccination rates and prognoses of patients receiving inpatient treatment with the diagnosis of Covid-19 in our pandemic clinics were investigated. 316 patients who were followed up in the clinic were examined. Among these patients, 77 (24.4\%) patients were found to be vaccinated. It was determined that 239 patients $(75.6 \%)$ were not vaccinated. The mean age was $41.6 \pm 5$ in vaccinated patients and $24.4 \pm 4$ in unvaccinated patients. While being followed in the clinic, 65 of the 78 patients who were taken to the intensive care unit were unvaccinated and 13 were vaccinated. No one died among those who were vaccinated. As a result of our study, it was determined that the vaccine reduced the rate of hospitalization significantly, similar to other studies. In addition, it has been observed that the vaccine has positive effects on the mortality and morbidity of the patients followed in the intensive care unit.
\end{abstract}

Keywords: Covid-19, pandemic, vaccine, mortality, morbidity

\section{Introduction}

Covid-19 infection was detected for the first time in January 2020 and subsequently became a pandemic. Covid-19 infection has also shown how fragile public health is in the face of epidemics. It is predicted by the scientific world that the most effective way to combat this epidemic is vaccination (World Health Organization, 2020).

i Correspondence: email esra.arslanaksu@saglik.gov.tr 
Studies on the vaccine, which is the most effective method against the virus, which spreads rapidly all over the world and causes many deaths, also gained momentum in this process. As of 31 December 2020, publications on the efficacy and safety of several vaccines have begun (Voysey et al., 2021). Phase 3 studies of other vaccines are still continuing (Hodgson et al., 2021). It was stated by the World Health Organization (WHO) and U.S. Food and Drug Administration (FDA) that the developed vaccines are $90 \%$ effective in terms of the emergence of the symptoms of the disease and the transmission of severe disease (Corey et al., 2020). These vaccines have been approved for immediate use by the FDA.

The first case in Turkey was detected in March 2020. Community vaccination was launched across the country in January 2021. Vaccination process started with healthcare professionals, individuals over 65 years of age and those with chronic diseases, and the area was expanded in stages. Vaccination strategies have been created with similar approaches in the world (Biggs and Littlejohn, 2021).

\section{Material and Method}

Patients who received inpatient treatment with the diagnosis of Covid-19 in the pandemic clinic of our hospital between March-April-May 2021 were examined. Patients with a positive PCR test and a diagnosis of Covid-19 with hospitalization indication were included in the study. Patients who were found to be incidentally positive for PCR (preoperative evaluation, routine PCR test positivity from patients who were planned to be hospitalized for another reason, etc.) patients whose radiological appearance was suspicious for viral pneumonia and those whose PCR results were negative twice were not included in the study.

The data of the patients were obtained by retrospectively scanning the files and examining the database data. Patients' age, vaccination status, time between vaccination and hospitalization, and comorbidities were evaluated. Vaccination status of patients who were transferred to the intensive care unit while hospitalized in the clinic was also examined. In the light of the data obtained, results were found on vaccination rates and clinical follow-ups in hospitalized patients.

\section{Results}

In the pandemic clinics of Samsun Training and Research Hospital, 316 patients who met the inclusion criteria in March-April-May 2021 were examined. Among these patients, 77 $(24.4 \%)$ patients were found to be vaccinated. It was determined that 239 patients $(75.6 \%)$ were not vaccinated (Figure 1).

The mean age of the vaccinated patients was $41.6 \pm 5$ years. It was found that 44 $(57.1 \%)$ of these patients had chronic diseases. The most common chronic diseases were hypertension and diabetes mellitus.

The mean age of the unvaccinated patients was $24.4 \pm 4$ years. It was observed that $29(12.1 \%)$ of the unvaccinated patients had a chronic disease and $210(87.9 \%)$ patients 
did not have a chronic disease. The most common chronic diseases in patients who were not vaccinated were hypertension and diabetes mellitus.

When 78 patients were admitted to the intensive care unit due to increased respiratory distress after making a physical examination in the clinic, it was found that $65(83.3 \%)$ of these patients were unvaccinated.

The mean age of the patients who were unvaccinated and transferred to the intensive care unit was $60.6 \pm 3.31(47.7 \%)$ of the patients who were transferred to the intensive care unit from the unvaccinated patients died during the intensive care followup. The mean age of the patients with exitus was $69.9 \pm 4$.

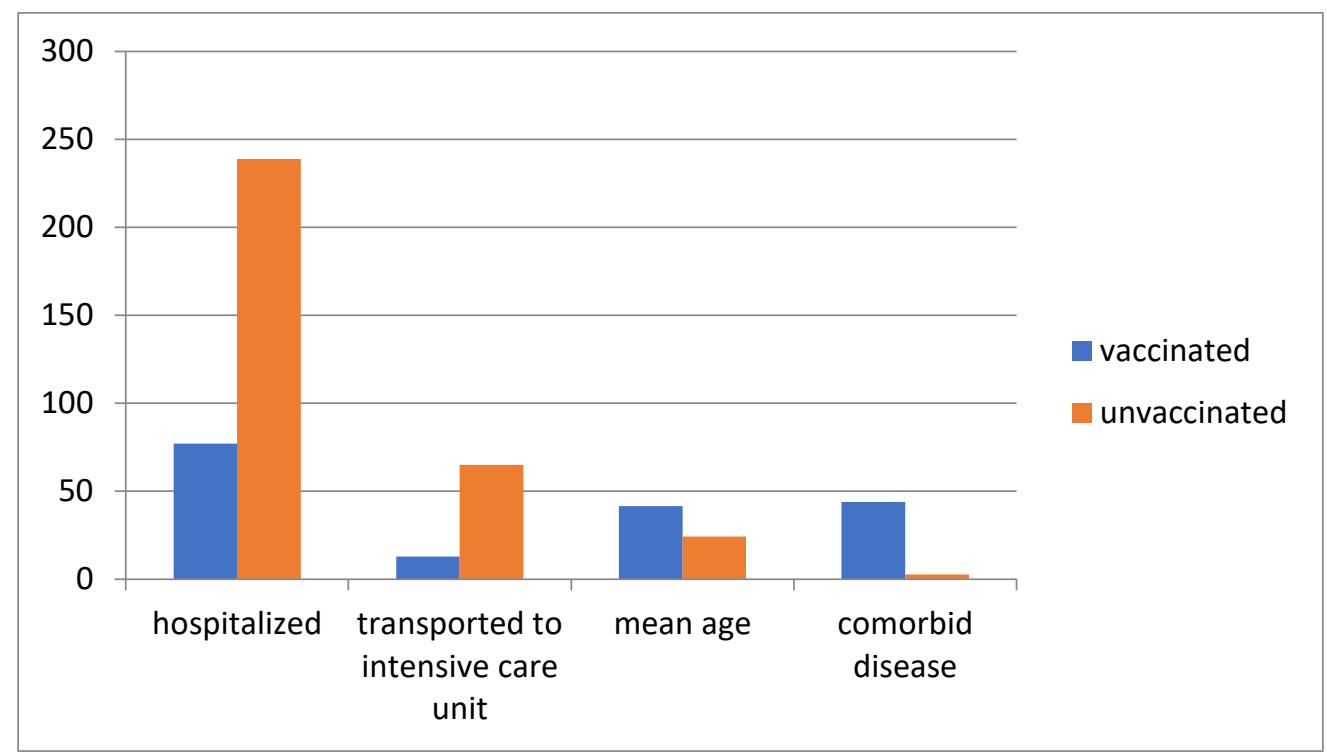

Figure 1: Data on vaccinated and unvaccinated patients hospitalized with the diagnosis of Covid-19

The mean age of 13 patients who were vaccinated and transferred to the intensive care unit was $73.3 \pm 5$ While being followed in the clinic, the vaccinated patients who were transferred to the intensive care unit did not die, and it was determined that all of these patients had comorbid diseases.

\section{Discussion}

The Covid-19 pandemic continues in our country and all over the world. Vaccines are seen as the only hope all over the world. However, an effective vaccination strategy must be implemented in order to achieve the target. It is predicted that a return to daily life can be possible with vaccination. As in the rest of the world, in our country, it was planned to start vaccination with people over 65 years of age, healthcare workers and individuals with chronic diseases and gradually vaccinate the entire population.

It can be seen that the vaccine plays an active role in coping with the epidemics seen in the world previously. Despite this, there are groups that are against the vaccine all over the world. Demonstrating the effect of the vaccine on Covid-19 infection is necessary for public health. This is only possible by conducting scientific studies and 
publishing the results. Studies have shown that the rates of hospitalization decreased in the vaccinated groups. There are studies showing that mortality rates are reduced by $90 \%$, especially in the elderly patient group, and that is $95 \%$ effective in preventing the disease (Garg et al., 2020).

In our study, when the patients who were transferred to the intensive care unit from in patients with the diagnosis of Covid-19 between March-April-May 2021 were examined, it was determined that there were no deaths in the vaccinated patients. All of the patients who were vaccinated and transferred to the intensive care unit had additional chronic diseases. Cardiovascular diseases, diabetes and hypertension were the most common comorbid diseases, similar to the other studies (Adams et al., 2020).

The majority of those hospitalized in the clinic who were not vaccinated consisted of individuals without any additional disease. In the vaccinated group, the proportion of individuals with additional diseases was higher. In previous studies, it has been shown that hospitalization rates are higher for those with one or more concomitant comorbid diseases (Carrillo-Vega et al., 2020).

When these results are evaluated, it can be concluded that the vaccine significantly reduces hospitalization in Covid-19 patients, especially in people who do not have any additional disease. The fact that there is no death in patients with chronic diseases who are vaccinated and transferred to the intensive care unit shows that the vaccine reduces mortality.

The mean age of the patients investigated in our study was $41.6 \pm 5$ in those who were vaccinated and $24.4 \pm 4$ in those who were not. The mean age was below 65 years in both groups. At the time the study was scheduled, the vaccination program was carried out in our country for individual over 50 years old, excluding healthcare workers. For this reason, it is though that the rate of hospitalization is low because individuals over the age of 50 are vaccinated and therefore this is reflected in our study in terms of average age.

According to our study, it was determined that vaccination against Covid-19 reduces the rate of hospitalization. The absence of a patient who died in the vaccinated group among the patients transferred to the intensive care unit showed that vaccination had positive effects on mortality as well as morbidity.

In our study, patients who received two doses of vaccine were evaluated. The time between vaccination and hospitalization was as long as the predicted active immunization period. Further studies on the necessity of booster doses will also be needed over time.

\section{Recommendations}

In line with the results found in the study, it can be recommended that everyone must be vaccinated against COVID-19.

COVID-19 will be with us for many years into the future. Getting vaccinated now has many benefits, including: protecting yourself against severe illness and death from COVID-19, preventing complications such as long COVID, protecting people who 
can't be vaccinated due to medical conditions, slowing the spread of the virus, keeping hospitalisation rates at a level our health system can cope with reducing the need for lockdowns in the future.

\section{Conflict of Interest Statement}

The author declare no conflicts of interests.

\section{About the Authors}

Dr. Esra Arslan Aksu is a doctor at Samsun Training and Research Hospital, Department of Chest Disease, Samsun, Turkey.

Ass. Prof. Demet Yalcin Kehribar is a doctor at Ondokuz Mayis University, Medical Faculty, Department of Internal Medicine, Samsun, Turkey.

\section{References}

Adams, M. L., Katz, D. L., \& Grandpre, J. (2020). Population-based estimates of chronic conditions affecting risk for complications from coronavirus disease, United States. Emerging infectious diseases, 26(8), 1831.

Biggs, A. T., \& Littlejohn, L. F. (2021). Revisiting the initial COVID-19 pandemic projections. The Lancet Microbe, 2(3), e91-e92

Carrillo-Vega, M. F., Salinas-Escudero, G., García-Peña, C., Gutiérrez-Robledo, L. M., \& Parra-Rodríguez, L. (2020). Early estimation of the risk factors for hospitalization and mortality by COVID-19 in Mexico. PLoS One, 15(9), e0238905.

Corey, L., Mascola, J. R., Fauci, A. S., \& Collins, F. S. (2020). A strategic approach to COVID-19 vaccine R\&D. Science, 368(6494), 948-950.

Garg, S., Kim, L., Whitaker, M., O’Halloran, A., Cummings, C., Holstein, R., \& Fry, A. (2020). Hospitalization rates and characteristics of patients hospitalized with laboratory-confirmed coronavirus disease 2019 COVID-NET, 14 States, March 130, 2020. Morbidity and mortality weekly report, 69(15), 458.

Hodgson, S. H., Mansatta, K., Mallett, G., Harris, V., Emary, K. R., \& Pollard, A. J. (2021). What defines an efficacious COVID-19 vaccine? A review of the challenges assessing the clinical efficacy of vaccines against SARS-CoV-2. The lancet infectious diseases, 21(2), e26-e35.

Voysey, M., Clemens, S. A. C., Madhi, S. A., Weckx, L. Y., Folegatti, P. M., Aley, P. K., \& Bijker, E. (2021). Safety and efficacy of the ChAdOx1 nCoV-19 vaccine (AZD1222) against SARS-CoV-2: an interim analysis of four randomised controlled trials in Brazil, South Africa, and the UK. The Lancet 397(10269): 99-111.

World Health Organization. (2020). Coronavirus disease (COVID-2019) press briefings. Geneva: World Health Organization. 
Author(s) will retain the copyright of their published articles agreeing that a Creative Commons Attribution 4.0 International License (CC BY 4.0) terms will be applied to their work. Under the terms of this license, no permission is required from the author(s) or publisher for members of the community to copy, distribute, transmit or adapt the article content, providing a proper, prominent and unambiguous attribution to the authors in a manner that makes clear that the materials are being reused under permission of a Creative Commons License. Views, opinions and conclusions expressed in this research article are views, opinions and conclusions of the author(s). Open Access Publishing Group and European Journal of Public Health Studies shall not be responsible or answerable for any loss, damage or liability caused in relation to/arising out of conflicts of interest, copyright violations and inappropriate or inaccurate use of any kind content related or integrated into the research work. All the published works are meeting the Open Access Publishing requirements and can be freely accessed, shared, modified, distributed and used in educational, commercial and non-commercial purposes under a Creative Commons Attribution 4.0 International License (CC BY 4.0). 